\title{
Siglorum et compendiorum explicatio
}

\begin{tabular}{|c|c|}
\hline$\langle\alpha \alpha \alpha\rangle$ & litterae coniectura additae \\
\hline$[\alpha \alpha \alpha]$ & litterae coniectura restitutae \\
\hline$\llbracket \alpha \alpha \alpha \rrbracket$ & litterae a librario deletae \\
\hline$(\alpha \alpha \alpha)$ & litterae per compendium a librario omissae \\
\hline$\{\alpha \alpha \alpha\}$ & litterae delendae \\
\hline$\alpha \alpha \underline{\alpha} \alpha$ & litterae valde incertae \\
\hline$[\ldots]$ & numerus litterarum quae perierunt \\
\hline ]...[ & litterarum vestigia dubia \\
\hline$\llcorner\alpha \alpha \alpha\lrcorner$ & litterae ex testimonio alio antiquo allatae \\
\hline I & versus finis \\
\hline add. & addidit, addiderunt \\
\hline adesp. & adespotum, adespota \\
\hline ad 1. & ad locum \\
\hline agn. & agnovit, agnoverunt etc. \\
\hline ap. & apud \\
\hline app. & apparato \\
\hline app. crit. & apparato critico \\
\hline app. pap. & apparato papirologico \\
\hline ca. & circa \\
\hline cet. & ceteri \\
\hline cf. & confer \\
\hline cfr. & confronta \\
\hline cit. & citazione \\
\hline cl. & collato \\
\hline codd. & codices \\
\hline col., coll. & colonna, colonne \\
\hline coni. & coniecit, coniecerunt etc. \\
\hline $\mathrm{mm}$ & commento \\
\hline
\end{tabular}


del.

delevit, deleverunt

dub.

dubitanter

ed., edd.

editio, edidit, editor, editores

ed. pr.

editor, editio princeps

e.g.

exempli gratia

es.

esempio

fort.

fortasse

fr., frr.

frammento, frammenti

init.

initium

inv.

inventario

mg.

margine

n., nn.

nota, note

nr., nrr.

numero, numeri

p., pp.

pagina, pagine

pap.

papiro

p.c.

post correctionem

poss.

possibile, possis

pot.

potius

prob.

probavit, probaverunt

r., rr.

rigo, righi

s., ss.

seguente, seguenti

sc.

scilicet

sch.

scholium, scholia

saec.

saeculum

simm.

similia

sup.

superiore

suppl.

supplevit, suppleverunt, etc.

susp.

suspicans, suspicatur, suspicantur etc.

s.v., s. vV.

sub voce, sub vocibus

tempt.

temptavit

V., vV.

verso, versi

vd.

vedi

veri sim.

veri simile, verisimiliter

vol.

volume 